\title{
Continuous Quality Improvement of Radiation Safety Management for Treatment of Differentiated Thyroid Carcinoma with lodine-131
}

\author{
Miaoli Zhou ${ }^{1}$, Qingran Lin ${ }^{2}$, Jinmei Xiong ${ }^{1}$, Lijiao Liao ${ }^{1}$, Chunliu Luo ${ }^{3}$, Jian Gong ${ }^{1}$, Hao Xu$^{1}$, * \\ ${ }^{1}$ Department of Nuclear Medicine, The First Affiliated Hospital of Jinan University, Guangzhou, China \\ ${ }^{2}$ Nursing Department, The First Affiliated Hospital of Jinan University, Guangzhou, China \\ ${ }^{3}$ The Image Center, The First Affiliated Hospital of Jinan University, Guangzhou, China \\ Email Address: \\ 13622898846@163.com (Miaoli Zhou), qingranlin@126.com (Qingran Lin), 190598691@qq.com (Jinmei Xiong), \\ 272982941@qq.com (Lijiao Liao),jnuchll@163.com (Chunliu Luo), dianchi555@sina.com (Jian Gong), hao_xu 2019@163.com (Hao Xu) \\ ${ }^{*}$ Corresponding author
}

\section{To cite this article:}

Miaoli Zhou, Qingran Lin, Jinmei Xiong, Lijiao Liao, Chunliu Luo, Jian Gong, Hao Xu. Continuous Quality Improvement of Radiation Safety Management for Treatment of Differentiated Thyroid Carcinoma with Iodine-131. American Journal of Nursing Science.

Vol. 9, No. 3, 2020, pp. 120-123. doi: 10.11648/j.ajns.20200903.17

Received: March 31, 2020; Accepted: April 13, 2020; Published: April 28, 2020

\begin{abstract}
Purpose: To explore the effect of continuous quality improvement (CQI) of radiation safety management for patients administerediodine-131 after thyroid cancer surgery. Methods: A total of 103 patients with differentiated thyroid carcinoma were randomly divided into control and experimental groups containing 51 and 52 patients, respectively. In the control group, the drug was administered according to the operating procedure for iodine-131 treatment of thyroid carcinoma. In the experimental group, CQI was adopted to manage the radiation safety of care in addition to the conventional iodine-131 thyroid cancer treatment procedures. We also improved radiation safety protection measures prior to drug administration, developed a flow chart of drug administration for patients, established a patient preview of the drug administration process, and enhanced health education and psychological intervention for the patient. Results: The environmental radiation around the drug delivery window was reduced $(P<0.05)$, total duration of exposure to the radiation was shortened $(P<0.05)$, drug drop rate was decreased to $0 \%$, and patient satisfaction was improved in the experimental group compared to the control group. Conclusions: Application of CQI for management of radiation safety when treating thyroid cancer withiodine-131 can improve patient treatment, quality of care, and satisfaction and reduce radiation pollution in the surrounding environment and radiation injury to staff.
\end{abstract}

Keywords: Differentiated Thyroid Carcinoma, Iodine-131-Administered Care, Radiation Safety Management,

Continuous Quality Improvement

\section{Introduction}

The iodine-131 is widely used in the treatment of differentiated thyroid carcinoma [1-3]. According to the Law of the People's Republic of China on the Prevention and Control of Radioactive Pollution and the Regulations on the Safety and Protection of Radioisotopes and Radiation Devices, when radiopharmaceuticals are employed, radiation protection and safety issues should be considered in addition to the chemical and biological properties of the drug [2]. Supervision of radioactive source and device safety in hospitals should be strengthened, radiation safety accidents should be reduced, the ability to respond to radiation accidents should be improved, and the consequences of accidents should be controlled and mitigated. Despite the clinical importance of measuring iodine deficiency, commonly used indicators have a number of limitations in their ability to reliably assess current iodine status. Urinary iodine concentration (UIC) from spot urine reflects recent die tartare intake (hours to days) and can have high daily variability $[4,5]$.

According to the routine regulations during the treatment 
of patients with thyroid cancer by administration of iodine-131, The radioactive liquid spill would be occurred frequently. In addition, the radiation levels around the delivery window were high after the drug was taken, which affected the patient quality of treatment/care and the environmental safety of the hospital. In addition, studies reporting iodine-131 is beta and gamma emitter and its special radiation in inducing damage is the beta particles. Beta particle irradiation, in comparison with $6 \mathrm{MV}$ X-ray, has advantages in the cellular damage. However, in addition to create self-dose in the surrounding cells, iodine-131 transfers a cross dose to adjacent and distant cells which increases DNA strand breaks $[6,7]$.

To this end, our nursing unit set up a quality improvement team starting in June 2016 to apply continuous quality improvement (CQI) to clinical practice activities in response to radiation safety problems in iodine-131 administration and achieved satisfactory results as described below. The objective of our study is to assess the influence of Continuous quality improvement of radiation safety management for treatment of differentiated thyroid carcinoma with iodine-131.

\section{Materials and Methods}

\subsection{Participants Enrollment and Study Methods}

103 patients with differentiated thyroid cancer that was treated with iodine-131 in our hospital's nucleus ward were enrolled in this study, and divided randomly into control and experimental groups. There were 51 cases in the control group consisting of 14 males and 37 females with an average age of $42.0 \pm 14.6$ years old. There were 52 patients in the experimental group consisting of 15 males and 37 females with an average age of $37.6 \pm 9.3$ years. In research, the exposure duration to the radioactive source in the control area was measured by stopwatch and the self-perception and cognitive status of the patient before taking the medication was assessed using a self-designed questionnaire.

Both groups of patients completed the relevant examination within the expected time and were treated with an oral sodium iodide-131 solution at a dose of 100-150 mCi. The control group patients receive the routine thyroid cancer treatment protocols in our study. On the other hand, we provide the CQI management to the experimental group patients. The CQI team consisted of nursing director, nursing team leader, and responsible nurse. The nursing director was responsible for technical guidance, the nursing team leader was responsible for project implementation and quality control, and the responsible nurse was in charge of recording group activity.

Inclusion criteria: patients (1) being treated with iodine-131 for differentiated thyroid cancer, (2) being treated with iodine-131 to clear residual thyroid tissue, (3) having normal cognitive and behavioral ability, and (4) providing informed consent for and being of stable condition during this study. Exclusion criteria: patients having (1) difficulty swallowing, (2) a physical activity disorder, (3) severe anxiety or depression, (4) severe damage to the oral mucosa, (5) received iodine-131 treatment of differentiated thyroid cancer more than once, and (6) severely impaired liver and kidney function.

\subsection{Statistical Method}

Statistical analysis was performed using SPSS 17.0 software. The data is presented as mean \pm standard deviation. Wilcoxon rank-sum tests were used to compare groups. The count data were analyzed by $\chi^{2}$ test. A difference was considered statistically significant at $P<0.05$.

\section{Results}

\subsection{Comparison of Radiation Monitoring Values After Patients Were Administered the Drugs}

After the CQI principle was applied for radiation safety management, the difference in radiation monitoring values between the two groups was statistically significant $(P<0.05)$, as shown in Table 1.

\subsection{Comparison of Self-perceived Patient Cognition Before Taking Drugs}

The self-perception cognition of the two groups before drug administration was assessed and the experimental group was found to have superior self-perceived cognition compared to the control group. The difference was statistically significant $(P<0.05)$, as shown in Table 2 .

\subsection{Comparison of Patient Satisfaction of the Explanation of the Medication Process and Implementation Methods}

By comparing the satisfaction of the two groups concerning the medication administration process and implementation method, it was found the experimental group was statistically significantly $(P<0.05)$ more satisfied than the control group, as shown in Table 3.

Table 1. Comparison of radiation monitoring after patient took drugs (Wilcoxon rank-sum test for comparison of raw data for two independent samples).

\begin{tabular}{llll}
\hline Group & Number of patients & $\begin{array}{l}\text { Radiation monitoring for environment surrounding the } \\
\text { dosing window (CPM) }\end{array}$ & $\begin{array}{l}\text { Duration of exposure to radiation } \\
\text { source (S) }\end{array}$ \\
\hline Control & 51 & 1879.2 & 465.62 \\
Test & 52 & 433.82 & 272.82 \\
$Z$ value & & 1.921 & 4.389 \\
P value & & 0.001 & 0.000 \\
\hline
\end{tabular}


Table 2. Comparison of patient self-perceived cognition before taking drug.

\begin{tabular}{|c|c|c|c|c|c|c|}
\hline \multirow{2}{*}{ Item } & \multicolumn{2}{|c|}{ Control $(n=51)$} & \multicolumn{2}{|c|}{ Test $(n=52)$} & \multirow{2}{*}{$\mathrm{X}^{2}$ value } & \multirow{2}{*}{ P value } \\
\hline & Yes & No & Yes & No & & \\
\hline Not adapted to the hospital environment & $33(64.7)$ & $18(35.3)$ & $25(48.1)$ & $27(51.9)$ & 2.894 & 0.089 \\
\hline Mental tension before taking the medicine & $36(70.6)$ & $15(29.4)$ & $12(23.1)$ & $40(76.9)$ & 23.356 & 0.000 \\
\hline Worry about spillage when taking the medicine process & $35(68.6)$ & $16(31.4)$ & $17(32.7)$ & $35(67.3)$ & 13.301 & 0.000 \\
\hline Familiar with medication process & $36(70.6)$ & $15(29.4)$ & $51(98.1)$ & $1(1.9)$ & 12.807 & 0.000 \\
\hline Requirement of pre-medication training rehearsal & $47(92.2)$ & $4(7.8)$ & $49(94.2)$ & $3(5.8)$ & 0.001 & 0.979 \\
\hline Flow chart of medication helps & $47(92.2)$ & $4(7.8)$ & $51(98.1)$ & $1(1.9)$ & 0.882 & 0.348 \\
\hline
\end{tabular}

Table 3. Comparison of patient satisfaction of the medication process explanation and implementation methods (comparison of sample composition ratio).

\begin{tabular}{|c|c|c|c|c|}
\hline Group & Number of patients & Very satisfied & Satified & Unsatified \\
\hline Control & 51 & 16 & 35 & 0 \\
\hline Test & 52 & 39 & 13 & 0 \\
\hline $\mathrm{X}^{2}$ Value & - & - & 19.694 & \\
\hline
\end{tabular}

\section{Discussion}

The key point of Continuous Quality Improvement (CQI) is focused on supervising and taking good care of patients during the quality improvement process, thus continuously improving the results [4]. Due to the widespread use of radionuclide iodine-131 for the treatment of thyroid cancer, related nursing processes and methods continue to advance and develop. Radiation safety management ofiodine-131 administration is a topic of particular interest to many scholars in the clinic. Iodine-131 emits a gamma ray and, thus, is highly penetrating, is volatile, and has a long half-life. A lead plate is therefore needed as a shield to prevent iodine-131 penetration. If the radiation protection measures are improperly implemented during the administration to thyroid cancer patients, leakage of the liquid will not only inhibit decontamination of the staff, but also pollute the indoor air and surrounding environment $[8,9,10]$. At the same time, when a patient takes the drug and becomes a strong "radiation source", they should stay in the control area for a longer period to decrease the probability of environmental pollution. The nursing unit dosing window is connected to the ward corridor. Although the ward corridor is the control area, it is also used for staff to treat and care for the patient. According to radiation safety management regulations, the radiation dose that the staff receives should be minimized $[11,12]$. Combined research indicates that time, distance, shielding, and other protective measures are the most beneficial to avoiding exposure to radiation [13-15]. Additionally, high thyroid volume is an indicator of long-term iodine deficiency that may persist for years after iodine repletion [16].

The results of CQI measures in this study showed the total duration of the patient medication administration was shortened and the radiation pollution value of the ground around the drug administration window was reduced. Of course, if the ambient radiation monitoring value was higher than $0.5 \mu \mathrm{Sv} / \mathrm{hr}(167 \mathrm{CPM})$, the second layer of anti-seepage adhesive paper was replaced to reduce the ambient radiation monitoring value and keep the area safe, thus confirming CQI measures could not only reduce the probability of environmental pollution, but also reduce the workload of and risk of radiation damage to the staff, thus playing a very important role in radiation safety management.

The International Committee on Radiological Protection (ICRP) suggests that the medical staff should take appropriate protective measurements since the radiation dose caused by ${ }^{131} \mathrm{I}$ is the largest among medical staff, relatives and caregivers. [8] This study showed that, via a series of improvements, the risk of care was reduced and no adverse events occurred. However, the following deficiencies still remain. (1) Patients who indicate they are not suitable for the drug administration environment may have an inability to adapt to changes in their living environment and the restriction of free going out after taking the medicine. (2) The psychological tension and worry about drug leakage by the patients before taking the drug may be related to the instructor using the whole-process monitoring video method during medication administration rather than providing face-to-face and hand-to-hand guidance for taking the drug. Meanwhile, factors, such as economy or treatment effect, can also cause patient psychological responses, such as tension and fear. (3) The patient proficiency level in the medication process may be related to limited language communication and hypothyroidism, which can also have certain effects on cognitive function. Therefore, we will continue to actively explore effective nursing measures for these patients while strengthening health education and psychological interventions in the future.

In conclusion, we have adopted the CQI method for routine iodine-131 administration for thyroid cancer and achieved positive results in radiation safety management of radiopharmaceutical administration. We ensured the accuracy of patient dose, improved the quality of and patient satisfaction in the treatment, and reduced unnecessary radiation damage of the staff. Therefore, the CQI method is worth promoting and implementing. 


\section{References}

[1] Bilek R, Cerovska J, Zamrazil V. The relationship between iodine intake and serum thyroglobulin in the general population. Physiol. Res. 2015; 64 (3): 345-353.

[2] Haugen BR, Alexander EK, Bible KC, Doherty GM, Mandel SJ, Nikiforov YE, et al. 2015 American Thyroid Association Management Guidelines for Adult Patients with Thyroid Nodules and Differentiated Thyroid Cancer: the American Thyroid Association Guidelines Task Force oBilek R, Cerovska J, Zamrazil V. The relationship between iodine intake andserum thyroglobulin in the general population. Physiol. Res. 2016; 64 (3): 345-353.

[3] Lauri C, Di Traglia S, Galli F, et al. Current status of PET imaging of differentiated thyroid cancer with second generation radiopharmaceuticals. $Q J$ NuclMed Mol Imaging 2015, 59, 105-115.

[4] Ma ZF, Venn BJ, Manning PJ, Cameron CM, Skeaff SA. Iodine supplementation of mildly iodine-deficient adults lowers thyroglobulin: a randomized controlled trial. J. Clin. Endocrinol. Metab. 2016; 101 (4): 1737-1744.

[5] Madureira D, Prazeres S, Pedro MS, Pereira T, Font AP, Bugalho MJ. Invitro assays to test the interference of anti-thyroglobulin antibodies on thyroglobulin measurement. Endocrine. 2018; 33 (1): 40-44.

[6] Trimboli P, Treglia G, Giovanella L. Preoperative measurement of serum thyroglobulin to predict malignancy in thyroid nodules: a systematic review. Horm. Metab. Res. 2015; 47 (4): $247-252$.

[7] Molinaro E, Giani C, Agate L, Biagini A, Pieruzzi L, Bianchi, $\mathrm{F}$, et al. Patients with differentiated thyroid cancer who underwent radioiodine thyroid remnant ablation with low-activity ${ }^{131} \mathrm{I}$ after either recombinant human TSH or thyroid hormone therapy withdrawal showed the same outcome after a 10-year follow-up. J. Clin. Endocrinol. Metab. 2017; 98 (7): 2693-2700.

[8] Tonacchera M, Pinchera A, Dimida A, Ferrarini E, Agretti P,
Vitti P, et al. Relative potencies and additivity of perchlorate, thiocyanate, nitrate, and iodide onthe inhibition of radioactive iodide uptake by the human sodium iodide symporter. Thyroid. 2018; 14 (12): 1012-1019.

[9] D'Alessio D, Giliberti C, Benassi M, et al. Potential third-party radiation exposure from patients undergoing therapy with ${ }^{131} \mathrm{I}$ for thyroid cancer or metastases. Health Phys. 2015, 108, 319-325.

[10] Eyvazzadeh, Nazila, Neshasteh-Riz, Ali, Mahdavi, Seyed, Rabee, Mohsenifar, Afshin. Genotoxic damage to glioblastoma cells treated with $6 \mathrm{MV}$ X-radiation in the presence or absence of methoxy estradiol, IUDR or topotecan. Cell J. 2015; 17 (2): 312.

[11] Stezhko VA, Buglova EE, Danilova LI, Drozd VM, Krysenko NA, Lesnikova NR, et al. A cohort study of thyroid cancer and other thyroid diseases after the Chornobyl accident: objectives, design and methods. Radiat. Res. 2018; 161 (4): 481-492.

[12] Thomson CD, Woodruffe S, Colls AJ, Joseph J, Doyle TC. Urinary iodine and thyroid status of New Zealand residents. Eur. J. Clin. Nutr. 2016; 55 (5): 387-392.

[13] Pedersen IB, Laurberg P, Knudsen N, Jorgensen T, Perrild H, Ovesen L, et al. Smoking is negatively associated with the presence of thyroglobulin autoantibody and to a lesser degree with thyroid peroxidase autoantibody in serum: apopulation study. Eur. J. Endocrinol. 2018; 158 (3): 367-373.

[14] Likhtarov I, Kovgan L, Masiuk S, Talerko M, Chepurny M, Ivanova $\mathrm{O}$, et al. Thyroid cancer study among Ukrainian children exposed to radiation after theChornobyl accident: improved estimates of the thyroid doses to the cohort members. Health Phys. 2019; 106 (3): 370-396.

[15] Krejbjerg A, Bjergved L, Bulow Pedersen I, Carle A, Knudsen $\mathrm{N}$, Perrild H, et al. Serum thyroglobulin as a biomarker of iodine deficiency in adult populations. Clin. Endocrinol. 2016; $85,475-482$.

[16] Heemstra KA, Liu YY, Stokkel M, Kievit J, Corssmit E, Pereira AM, et al. Serum thyroglobulin concentrations predict disease-free remission and death in differentiated thyroid carcinoma. Clin. Endocrinol. 2017; 66 (1): 58-64. 